\title{
PHYLLODULCIN PROTECTS PC12 CELLS AGAINST THE INJURY INDUCED BY OXYGEN AND GLUCOSE DEPRIVATION-RESTORATION
}

\author{
CHANG-LONG HU', LU GE², YONG TANG ${ }^{1}$, JIE LI', CHUN-HUI WU', \\ JIANG-HONG HU' ${ }^{2}$ JIN-TAO YUAN ${ }^{1}$ and YONG-ZHONG FAN ${ }^{1 *}$
}

\begin{abstract}
'Department of Neurosurgery, ${ }^{2}$ Department of Gastroenterology, The People's Hospital of Danyang, Danyang, Jiangsu 212300, China
\end{abstract}

\begin{abstract}
Phyllodulcin is a natural coumarin derivative found in Hydrangea macrophylla. To discover effective therapeutics for cerebral ischemia-reperfusion injury, we investigated the protective effects of phyllodulcin on PC12 cells injury induced by oxygenation and glucose deprivation/restoration (OGD/R). As a result, phyllodulcin can improve the cell viability and attenuate the extracellular lactate dehydrogenase release. Meanwhile, phyllodulcin can ameliorate the dysfunction of mitochondria by reducing reactive oxygen species production, decreasing the intracellular calcium level and increasing the mitochondrial membrane potential. ROS-associated OGD/R can induce cell apoptosis through the mitochondrial pathway. Phyllodulcin can inhibit apoptosis of PC12 cells through down-regulation of Caspase- 3 and Bax as well as up-regulation of Bcl-2. These results indicate phyllodulcin can protect PC12 cells against the damage induced by OGD/R. This investigation gives promising evidences for the therapy of cerebral ischemia-reperfusion injury.
\end{abstract}

Keywords: phyllodulcin, PC12 cells, oxygen and glucose deprivation/restoration, apoptosis, oxidative stress

Cerebral ischemia-reperfusion injury is a leading cause of long term disability and death all over the world (1). The embolism of blood flow in the brain will lead to the deprivation of oxygen and glucose as well as the following brain tissue injury, then the restoration of oxygen and glucose will exacerbate the neuronal injury (2). Pathogenesis of cerebral ischemia-reperfusion includes oxidative stress, apoptosis and so on $(3,4)$. Oxidative stress plays an important role in the process of cerebral ischemiareperfusion (5). Cerebral ischemia-reperfusion can directly affect the function of mitochondria and break the metabolic homeostasis to induce oxidative stress for brain is susceptible to ischemic hypoxia (6). Reactive oxidative species (ROS) are produced in mitochondria and increased when the dysfunction of mitochondria and oxidative stress appear. The dysfunction of mitochondria will not only result in the overproduction of ROS but also an overload of intracellular calcium followed by neuronal apoptosis through the mitochondrial pathway (7-9). Meanwhile, LDH will be released into cells culture medium from cytoplasm when the cells die and the next cytoplasm membranes break (10). At present, few therapeutic approaches are available to cerebral ischemia-reperfusion injury for the complicated mechanisms. In the discovery of efficient therapeutics for cerebral ischemia-reperfusion injury, bioactive phytochemicals showed promising evidences such as aloin (10), acetylpuerarin (11), genistein (12) and so on.

Phyllodulcin (Fig. 1) is a natural coumarin derivative found in Hydrangea macrophylla as a natural sweetener (13). Previous pharmacological investigations have revealed phyllodulcin showed potential for regulating obesity (14), sensitizing cancer chemotherapy (15), relieving diabetes (16), blocking degranulation of mast cells (17), inhibiting phosphodiesterase (18) and suppressing fungus (19).<smiles>COc1ccc([C@H]2Cc3cccc(O)c3C(=O)O2)cc1O</smiles>

Figure 1. Chemical structure of phyllodulcin.

\footnotetext{
* Corresponding author: e-mail: fyzdy123@126.com
} 
In our program to search bioactive phytochemicals for the treatment of cerebral ischemia-reperfusion injury, we have evaluated the neuroprotective effects of phyllodulcin on PC12 cells induced by oxygen and glucose deprivation-restoration (OGD/R). Herein we report these effects and related mechanisms.

\section{MATERIALS AND METHODS}

\section{Chemicals and reagents}

Phyllodulcin was purchased from Yuanye Biotechnology Ltd. (Shanghai, China). 3-[4,5dimethylthiazol-2-yl]-2,5-diphenyltetrazolium bromide (MTT), and dimethyl sulfoxide (DMSO) were obtained from Sigma-Aldrich (St. Louis, MO, USA). Dulbecco's modified Eagle's medium (DMEM) was purchased from Invitrogen Gibco Co. (Grand Island, NY, USA). ROS, lactate dehydrogenase (LDH) and BCA assay kits were obtained from Nanjing Jiancheng Bioengineering Institute (Nanjing, China). Rhodamine-123, Fluo-3 AM and Caspase-3 assay kit as well as cleaved Caspase-3, $\mathrm{Bcl}-2$, Bax and $\beta$-actin antibodies were purchased from Beyotime Biotechnology Institute (Nantong, China).

\section{Cell culture and treatment}

PC12 cells (rat adrenal pheochromocytoma cells) were supplied by Cell Bank of Shanghai Institute of Biological Sciences, Chinese Academy of Sciences. The cells were cultured in DMEM with $10 \%$ fetal bovine serum, $1 \%$ penicillin and streptomycin under a humid $5 \% \mathrm{CO}_{2}$ and $95 \%$ air condition at $37^{\circ} \mathrm{C}$. To mimic the cerebral ischemia-reperfusion model in vitro, OGD/R in PC12 cells was carried out as previously described (20). In brief, the culture medium was replaced with glucose-free DMEM after washed. Then the cells were incubated in glucose-free DMEM at a $95 \% \mathrm{~N}_{2}$ and $5 \% \mathrm{CO}_{2}$ atmosphere with or without certain concentrations of phyllodulcin $(0.1,1$ and $10 \mu \mathrm{M})$ for $4 \mathrm{~h}$. And then the cells were cultured with DMEM in the absence or presence of phyllodulcin under a normal condition $\left(5 \% \mathrm{CO}_{2}\right.$ and $95 \%$ air) for $24 \mathrm{~h}$. The cells as control group were incubated in DMEM at normal atmosphere.

\section{MTT assay}

To evaluate the effect of phyllodulcin on PC12 cell viability, the MTT assay was employed. PC12 cells were added into 96-well microplates and adjusted to $1 \times 10^{5}$ per well. After treated as above, MTT was added into each well to be $0.5 \mathrm{mg} / \mathrm{mL}$ and the incubation was maintained for $4 \mathrm{~h}$ at $37^{\circ} \mathrm{C}$. Then the medium was removed and DMSO was used to dissolve the formazan. After $10 \mathrm{~min}$, the absorbance was measured at $570 \mathrm{~nm}$ on a microplate reader. Cell viability was expressed as a relative percentage of optical density (OD) values compared with the control group.

\section{LDH release assay}

The leakage of LDH in the extracellular matrix indicates the cells' injury (10). To determine the activity of LDH in PC12 cells culture medium, the LDH assay kit was employed. After treatment and incubation, $20 \mu \mathrm{L}$ supernatant in PC12 cells culture was sucked each time and treated according to the manufacturer's instructions. Then the absorbance was recorded on a microplate reader at $450 \mathrm{~nm}$. The activity of $\mathrm{LDH}$ can be calculated from the absorbance and expressed as U/L.

\section{Measurement of ROS}

The production of ROS was determined by the fluorescence method according to the manufacturer's instructions. After treatment, the medium was removed and the cells were washed with PBS. Then the 2',7'-dichlorodi-hydrofluorescein diacetate (DCFH-DA) in DMEM was added and incubation was implemented at $37^{\circ} \mathrm{C}$ for $30 \mathrm{~min}$. The cells were washed with PBS again and the fluorescence intensity was recorded on a fluorescence microplate reader at $485 \mathrm{~nm}$ (excitation wavelength) and $520 \mathrm{~nm}$ (emission wavelength).

\section{Measurement of intracellular calcium}

The level of intracellular calcium was determined by Fluo-3 AM molecular fluorescence probe according to the manufacturer's instruction. Briefly, pre-treated PC12 cells were incubated with Fluo-3 $\mathrm{AM}$ at $37^{\circ} \mathrm{C}$ for $1 \mathrm{~h}$ in dark, and then were washed with PBS for three times to remove the extracellular dye. The fluorescence was excited at $488 \mathrm{~nm}$ and emission wavelength was set at $525 \mathrm{~nm}$ on the fluorescence microplate reader.

\section{Determination of mitochondrial membrane potential}

Mitochondrial membrane potential (MMP) was also measured through a fluorescence probe, rhodamine-123. Rhodamine-123 can accumulate in mitochondria of normal cells, but it will be released into the cytosol and emit fluorescence while the MMP of apoptotic cells reduces (21). Following the treatment, $2 \mathrm{mM}$ rhodamine-123 was added into PC12 cells and incubated for $15 \mathrm{~min}$. Then the fluo- 
rescence intensity was read at an excitation wavelength of $488 \mathrm{~nm}$ and an emission wavelength of 530 $\mathrm{nm}$. MMP was expressed as the percentage of fluorescence intensity compared to the control group.

\section{Western blot analysis}

The PC12 cells were treated as the description in "cell culture and treatment" section and then subjected to Western blot analysis for the expression of
Caspase-3, Bcl-2, and Bax. In short, the cells were lysed with lysis buffer including $20 \mathrm{mM}$ Tris- $\mathrm{HCl}$ (pH 7.4), $150 \mathrm{mM} \mathrm{NaCl}, 1 \%$ Triton and $1 \mathrm{mM}$ phenylmethylsulfonyl fluoride (PMSF) on ice for 30 $\min$. Then the lysed cells were centrifuged at 12000 $\times \mathrm{g}$ and $4^{\circ} \mathrm{C}$ for $15 \mathrm{~min}$, and the supernatant was collected as the total protein for the analysis of cleaved Caspase-3, Bcl-2, and Bax. After quantified by a BCA assay kit, the samples were separated on $15 \%$

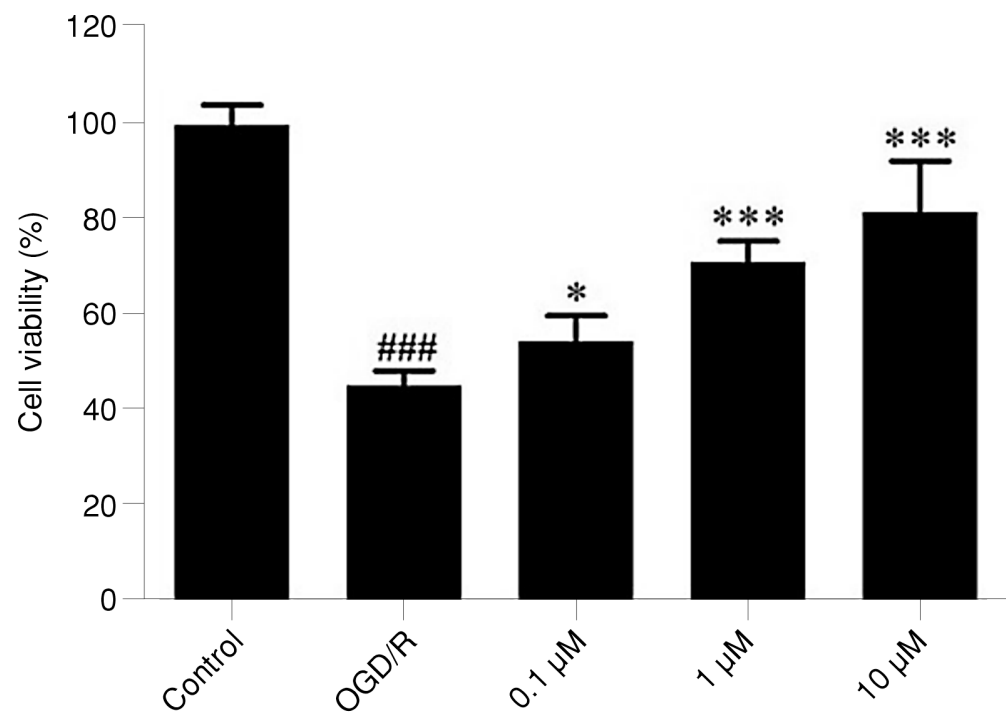

Figure 2. Effect of phyllodulcin on the PC12 cell viability. PC12 cells were treated by OGD with or without phyllodulcin $(0.1,1$ and 10 $\mu \mathrm{M}$ ) for $4 \mathrm{~h}$, and then exposed to $\mathrm{R}$ for $24 \mathrm{~h}$ in the presence or absence of phyllodulcin. The cell viability was performed by MTT assay. $\mathrm{n}=6$, \#\# $<0.001$ vs control group, ${ }^{*} \mathrm{p}<0.05,{ }^{* * *} \mathrm{p}<0.001$ vs OGD/R group.

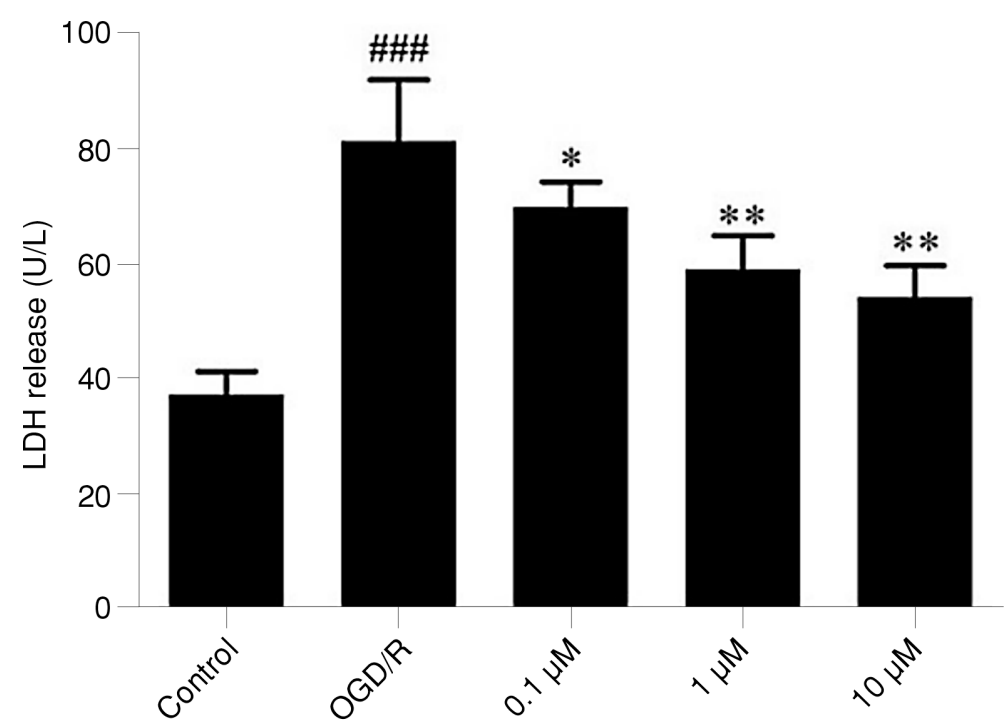

Figure 3. Effect of phyllodulcin on extracellular LDH. PC12 cells were treated by OGD with or without phyllodulcin $(0.1,1$ and $10 \mu \mathrm{M})$ for $4 \mathrm{~h}$, and then exposed to R for $24 \mathrm{~h}$ in the presence or absence of phyllodulcin. The activity of LDH in the supernatant of PC12 cells culture was determined by the LDH assay kit. $\mathrm{n}=6, \# \# \mathrm{p}<0.001$ vs control group, ${ }^{*} \mathrm{p}<0.05,{ }^{* *} \mathrm{p}<0.01$ vs OGD/R group. 
sodium dodecyl sulfate-polyacrylamide gel electrophoresis (SDS-PAGE) and transferred to polyvinylidene fluoride (PVDF) membranes. After blocked with $5 \%$ defatted milk, the membranes were incubated overnight with primary antibody of cleaved Caspase-3 (1 : 1000), Bcl-2 (1 : 1000), Bax $(1: 1000)$ and $\beta$-actin $(1: 1000)$ at $4^{\circ} \mathrm{C}$. The membranes were tested with respective secondary antibody conjugated to horseradish peroxidase at room temperature for $1 \mathrm{~h}$ and detected by enzyme-link chemiluminescence substrate. $\beta$-actin was used as internal control. Densitometric analysis was imple- mented by Image $\mathrm{J}$ software to obtain the relative intensities of bands.

\section{Caspase-3 activity}

The activity of Caspase- 3 was quantified by a colorimetric assay kit according to the manufacturer's instruction. The PC12 cells were pretreated as above and washed with PBS. Then they were lysed and centrifuged at $16000 \times \mathrm{g}$ and $4^{\circ} \mathrm{C}$ for $10 \mathrm{~min}$. The supernatant was incubated with substrate (AcDEVD- $p \mathrm{NA}$ ) at $37^{\circ} \mathrm{C}$ for $2 \mathrm{~h}$. The OD values were measured on a microplate reader at $405 \mathrm{~nm}$.

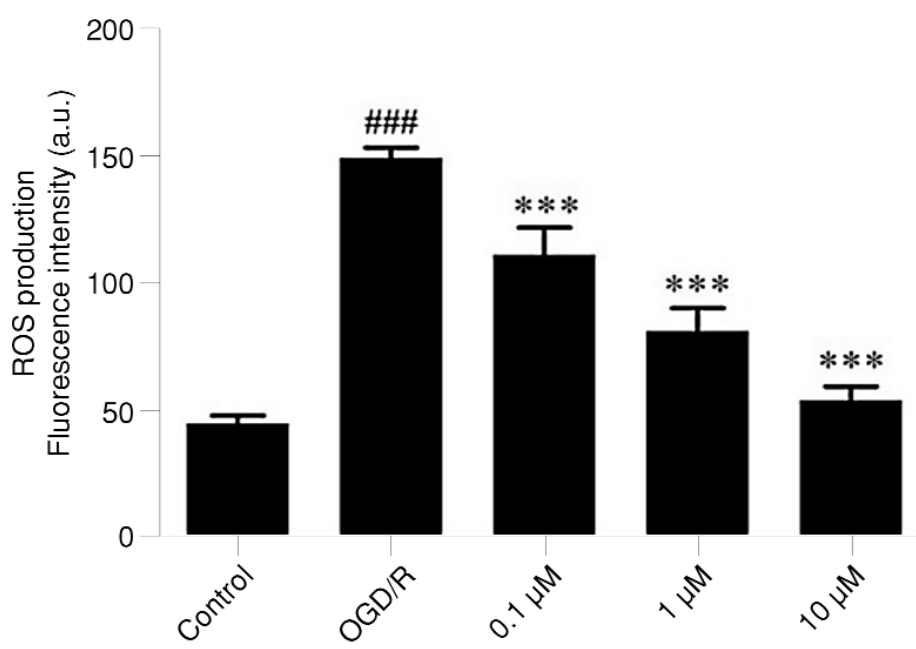

Figure 4. Effect of phyllodulcin on ROS production. PC12 cells were treated by OGD with or without phyllodulcin $(0.1,1$ and $10 \mu \mathrm{M})$ for $4 \mathrm{~h}$, and then exposed to $\mathrm{R}$ for $24 \mathrm{~h}$ in the presence or absence of phyllodulcin. The ROS production was presented as fluorescence intensity of hydrolyzed DCF. $\mathrm{n}=6, \# \# \mathrm{p}<0.001$ vs control group, $* * * \mathrm{p}<0.01$ vs OGD/R group.

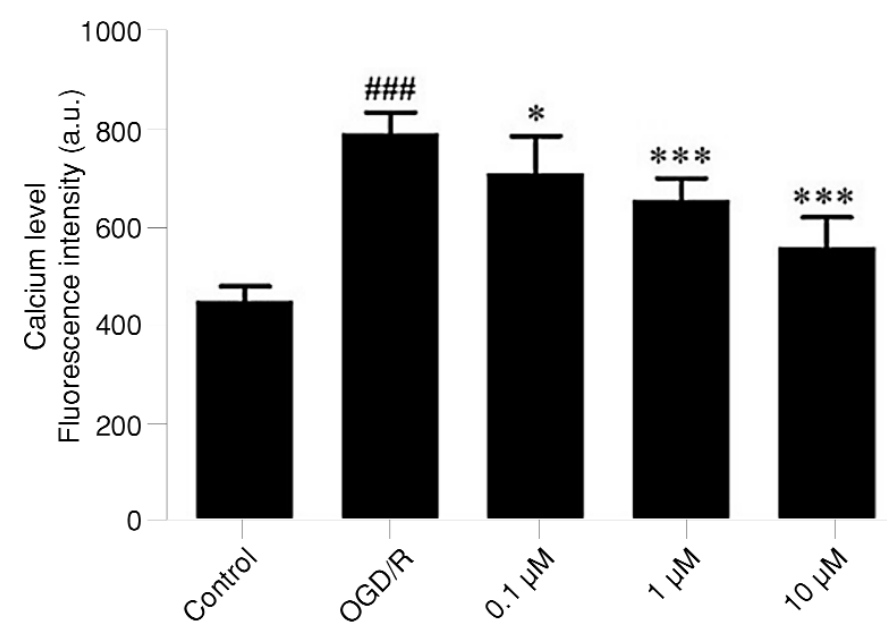

Figure 5. Effect of phyllodulcin on intracellular calcium. PC12 cells were treated by OGD with or without phyllodulcin $(0.1,1$ and $10 \mu \mathrm{M})$ for $4 \mathrm{~h}$, and then exposed to $\mathrm{R}$ for $24 \mathrm{~h}$ in the presence or absence of phyllodulcin. The intracellular calcium level was expressed as fluorescence intensity of Fluo-3 AM. $\mathrm{n}=6$, \#\#\# $<0.001$ vs control group, ${ }^{*} \mathrm{p}<0.05,{ }^{* * *} \mathrm{p}<0.001$ vs OGD/R group. 


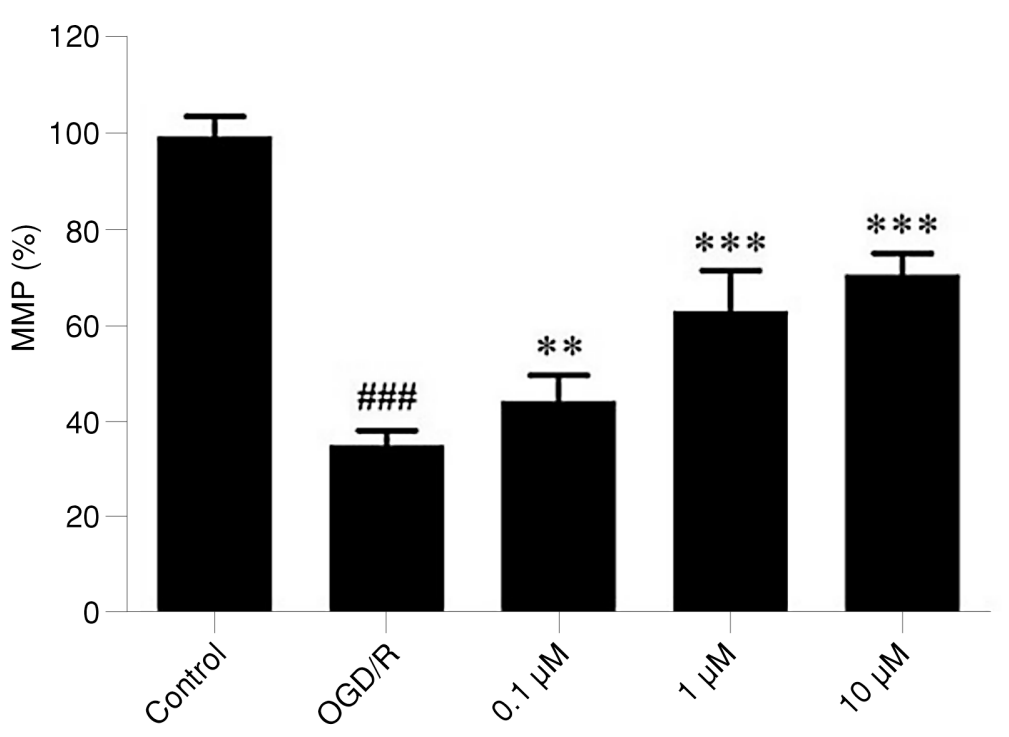

Figure 6. Effect of phyllodulcin on MMP. PC12 cells were treated by OGD with or without phyllodulcin $(0.1,1$ and $10 \mu \mathrm{M})$ for $4 \mathrm{~h}$, and then exposed to R for $24 \mathrm{~h}$ in the presence or absence of phyllodulcin. The MMP was expressed as the percentage compared with the control group. $\mathrm{n}=6, \# \# \mathrm{p}<0.001$ vs control group, $* * \mathrm{p}<0.01, * * * \mathrm{p}<0.001$ vs OGD/R group.

a)

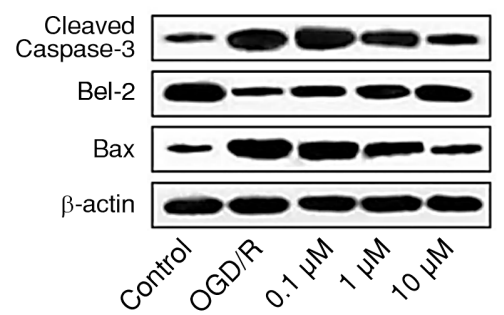

b)

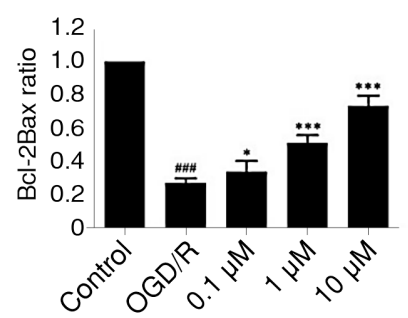

c)

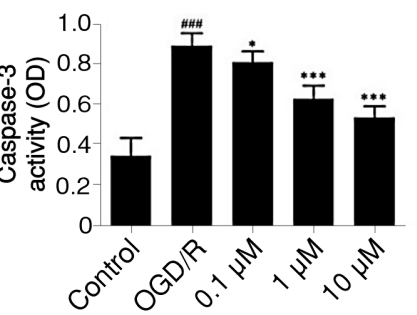

Figure 7. Effect of phyllodulcin on the apoptosis induced by OGD/R. (a) Western blot analysis of the expression of Caspase-3, Bcl-2 and Bax, (b) Densitometric analysis of the ratio between Bcl-2 and Bax derived from the Western blot, (c) The activity of Caspase-3 in PC1 2 cells, $\mathrm{n}=6, \# \# \mathrm{p}<0.001$ vs control group, $* \mathrm{p}<0.05, * * * \mathrm{p}<0.001$ vs OGD/R group.

\section{Statistical analysis}

All results were expressed as the means \pm standard deviation. GraphPad Prism was employed to analyze the results. Statistical differences between different groups were compared by one-way analysis of variance (one way ANOVA) followed by Tukey test for multiple comparisons and Student's t-test for single comparisons. And the difference was considered to be statistically significant when $\mathrm{p}<0.05$.

\section{RESULTS}

\section{Effect of phyllodulcin on cell viability}

The effects of phyllodulcin on PC12 cells viability were determined by MTT assay. As shown in
Fig. 2, after treatment by OGD/R, cell viability was markedly reduced $(45.55 \% \pm 3.05 \%(\mathrm{p}<0.001))$. When exposed to different concentrations of phyllodulcin, the cell viability was significantly increased in a dosage-dependent manner compared with the OGD/R group $(54.66 \% \pm 5.49 \%(\mathrm{p}<0.05), 71.39 \%$ $\pm 4.40 \%(\mathrm{p}<0.001)$ and $81.75 \% \pm 10.76 \%(\mathrm{p}<$ $0.001)$ ), which indicates phyllodulcin can attenuate the injury induced by OGD/R.

Effect of phyllodulcin on extracellular LDH

To further confirm the effect of phyllodulcin on the PC12 cells viability, the activity of LDH in the extracellular matrix was determined. After induction by OGD/R, the activity of LDH in the culture medium of $\mathrm{PC} 12$ cells increased to $81.75 \pm$ 
10.76 ( $\mathrm{p}<0.001)$ compared to the control group $(37.55 \pm 4.15 \mathrm{U} / \mathrm{L})$ (Fig. 3). But in the presence of phyllodulcin, the activity of the extracellular LDH was inhibited as $70.39 \pm 4.40 \mathrm{U} / \mathrm{L}(\mathrm{p}<0.05), 59.45$ $\pm 5.88 \mathrm{U} / \mathrm{L}(\mathrm{p}<0.01)$ and $54.66 \pm 5.49 \mathrm{U} / \mathrm{L}(\mathrm{p}<$ 0.01 ) according to the different concentrations. These results demonstrate phyllodulcin can prevent the death of PC12 cells affected by OGD/R.

\section{Effect of phyllodulcin on ROS production}

OGD/R can elevate the level of ROS in PC12 cells $(150.00 \pm 4.27, \mathrm{p}<0.001)$ by comparison with fluorescence intensity of control group (45.55 \pm 3.05) (Fig. 4). But the fluorescence intensity decreased to $111.75 \pm 10.76(\mathrm{p}<0.001), 81.72 \pm$ $9.13(\mathrm{p}<0.001)$ and $54.66 \pm 5.49(\mathrm{p}<0.001)$ following the treatment with phyllodulcin $(0.1,1$ and $10 \mu \mathrm{M})$. These results gave the evidence for that phyllodulcin can reduce the production of ROS.

\section{Effect of phyllodulcin on intracellular calcium}

After treatment with OGD/R, the concentration of intracellular calcium was increased through the fluorescence intensity $(800.00 \pm 42.67, \mathrm{p}<0.01)$ by contrast with the control group (455.54 \pm 30.50) (Fig. 5). But when exposure to different concentrations of phyllodulcin, significant reduction of fluorescence intensity $(717.52 \pm 76.99(\mathrm{p}<0.05)$, $663.90 \pm 44.04(\mathrm{p}<0.001)$ and $566.64 \pm 61.18(\mathrm{p}<$ $0.001)$ ) was detected, which demonstrates phyllodulcin can lessen the increased level of intracellular calcium in PC12 cells damaged by OGD/R.

\section{Effect of phyllodulcin on MMP}

The MMP was determined by the fluorescence probe rhodamine-123 to assess the depolarization of mitochondria for rhodamine-123 is proportional to MMP. As shown in Fig. 6, following the OGD/R, the MMP in PC12 cells was obviously reduced to $35.55 \% \pm 3.05 \%(\mathrm{p}<0.001)$. In contrast, when different concentrations of phyllodulcin at $0.1,1$ and $10 \mu \mathrm{M}$ appeared, the MMP in PC12 cells exhibited recovery with different extents as $44.66 \% \pm 5.49 \%(\mathrm{p}<0.01), 63.42 \% \pm 8.63 \%(\mathrm{p}<$ $0.001)$ and $71.06 \% \pm 4.58 \%(p<0.001)$. These results present the fact that phyllodulcin can ameliorate the dysfunction of mitochondria injured by $\mathrm{OGD} / \mathrm{R}$ in PC12 cells.

\section{Effect of phyllodulcin on Caspase-3, Bcl-2 and Bax}

The loss of MMP has been observed in PC12 cells injured by $\mathrm{OGD} / \mathrm{R}$, so the expression of Caspase-3, Bcl-2 and Bax were analyzed by West- ern blot to evaluate the anti-apoptotic effect of phyllodulcin. As a result, the expression of cleavedCaspase-3 and Bax was up-regulated after OGD/R. Accordingly, Bcl-2 was down-regulated (Fig. 7a). When different concentrations of phyllodulcin appeared in the culture system, the expression of cleaved Caspase-3 and Bax was down-regulated as well as Bcl-2 was up-regulated with different extents. As a criterion to measure apoptosis, the ratio between Bcl-2 and Bax derived from the densitometric analysis displayed phyllodulcin could inhibit apoptosis of PC12 cells exposed to OGD/R, since the ratio for $\mathrm{Bcl}-2 / \mathrm{Bax}$ was increased following the increment of phyllodulcin (Fig. 7b). The colorimetric assay further validated the effect of phyllodulcin on the activity of Caspase-3. From the Fig.7c, the absorbance in PC12 cells treated by $\mathrm{OGD} / \mathrm{R}(0.90 \pm 0.06, \mathrm{p}<0.001)$ was larger than that of control group $(0.36 \pm 0.09)$. With the addition of different phyllodulcin, the values were significantly reduced as $0.82 \pm 0.05(\mathrm{p}<0.05), 0.64 \pm 0.07(\mathrm{p}<$ $0.001)$ and $0.55 \pm 0.05$ ( $\mathrm{p}<0.001)$, respectively. This result demonstrates phyllodulcin can inhibit the activity of Caspase-3.

\section{DISCUSSION}

Cerebral ischemia-reperfusion injury is results from the OGD and restoration, which can induce neuronal apoptosis (5). PC12 cells treated by $\mathrm{OGD} / \mathrm{R}$ can mimic cerebral ischemia-reperfusion injury and be used to evaluate the potential effects of bioactive substances on that injury (10-12). Herein we assessed the protective effects of phyllodulcin on $\mathrm{PC} 12$ cells treated by OGD/R in vitro.

After exposure to $\mathrm{OGD} / \mathrm{R}$, the viability of PC12 cells was decreased, which demonstrated the cells' were injured. In the presence of phyllodulcin, the cells viability was increased and the injury was attenuated. LDH is a stable enzyme in the cytoplasm for energy metabolism. It will release into the culture medium when the plasma membrane is damaged. The activity of LDH in the culture supernatant indicates the viability of the cells. In this investigation, phyllodulcin can inhibit the leakage of LDH in the extracellular matrix induced by OGD/R.

Mitochondria is a crucial organelle affording energy metabolism and ROS production in living cells, and dysfunction of mitochondria is an early feature of ischemia in the nervous system (22). Ischemia-reperfusion can interrupt the homeostasis between the ROS generation and the detoxification and scavenging capacity of cellular antioxidant enzymes, which will lead to the oxidative stress in 
mitochondria and exacerbate the ischemia insult $(5,23)$. Calcium is a messenger in signal transduction and responsible for neurotransmitter release, modulation of membrane excitability and regulation of synaptic plasticity in the nervous system. The concentration of intracellular calcium is maintained through N-methyl-D-aspartic acid (NMDA) receptors and ion exchangers (24). Sustained elevated levels of calcium-induced by OGD/R may impair mitochondrial function and eventually lead to neuronal apoptosis (22). In addition, the overproduction of ROS can destroy the mitochondrial membrane through oxidative stress and result in the collapse of MMP (20). MMP is a sensitive indicator to characterize the function of mitochondria. Following the induction of apoptosis, the MMP is disrupted as one of the earliest intracellular events (21). Our results have shown the function of mitochondria in PC12 cells was disordered after OGD/R through the increasing ROS generation, elevated intracellular calcium level and decreasing MMP. While treated with phyllodulcin in advance, the dysfunction of mitochondria was significantly improved.

ROS-associated OGD/R can induce apoptosis through the mitochondrial pathway (5). Caspases are members of the cysteine-dependent aspartate proteases family and play an important role in cell apoptosis (25). Caspase-3 is one of the effector enzymes in apoptosis. Activation of Caspase- 3 is involved in ischemia through cleavage (26). Bcl-2 and Bax are members of the Bcl-2 protein family and play a pivotal role in the mitochondrion-derived apoptosis. Bcl-2 exhibits an anti-apoptotic effect and can attenuate the activation of Caspase- 3 while Bax shows a pro-apoptotic effect (27). In our investigation, the expression of activated Caspase- 3 and Bax in PC12 cells was up-regulated and Bcl-2 was down-regulated after OGD/R, which demonstrates the apoptosis induced by OGD/R occurs. On the contrary, in the presence of phyllodulcin, the expression of activated Caspase- 3 and Bax was down-regulated and Bcl-2 was up-regulated. The increased activity of Caspase- 3 affected by OGD/R was inhibited after the treatment of phyllodulcin. Moreover, the densitometric analysis for the ratio between Bcl2 and Bax also unravels the inhibitory effect of phyllodulcin on the apoptosis induced by OGD/R.

\section{CONCLUSION}

We investigated the protective effects of phyllodulcin on the PC12 cells against the injury induced by $\mathrm{OGD} / \mathrm{R}$ in vitro. Phyllodulcin can attenuate the PC12 cells damage through preventing oxidative stress and inhibiting ROS-mediated mitochondrial apoptosis. This investigation gives promising evidences for the therapy of cerebral ischemia-reperfusion injury.

\section{Acknowledgments}

This project was supported by Zhenjiang Key Research and Development Program (Social Development) (Grant No. SH2017033), Zhenjiang Social Development Directing Program (Grant No. FZ2017007), and Danyang Scientific Development Special Program (Grant No. SF201702) and the authors give sincere gratitude to those programs.

\section{Conflict of interest}

The authors declare no conflicts of interest.

\section{REFERENCES}

1. Krishnamurthi R.V., Moran A.E., Feigin V.L., Barker-Collo S., Norrving B. et al.: Neuroepidemiology 45, 190 (2013).

2. Hu Y., Deng H., Xu S., Zhang J.: Int. J. Mol. Sci. 16, 24895 (2015).

3. Saito A., Maier C.M., Narasimhan P., Nishi T., Song Y.S. et al.: Mol. Neurobiol. 31, 105 (2005).

4. Shi H.L., Liu K.J.: Front. Biosci. 12, 1318 (2007).

5. Mehta S.L., Manhas N., Raghubir R.: Brain Res. Rev. 54, 34 (2007).

6. Monteiro P., Oliveira P.J., Gonçalves L., Providência L.A.: Rev. Port. Cardiol. 22, 233 (2003).

7. Racay P., Tatarkova Z., Chomova M., Hatok J., Kaplan P., Dobrota D.: Neurochem. Res. 34, 1469 (2009).

8. Thompson J.W., Narayanan S.V., Koronowski K.B., Morris-Blanco K., Dave K.R., Perez-Pinzon M.A.: J. Bioenerg. Biomembr. 47, 101 (2015).

9. Granger D.L., Granger D.N.: J. Pathol. 190, 255 (2000).

10. Chang R., Zhou R., Qi X., Wang J., Wu F. et al.: Brain Res. Bull. 121, 75 (2016).

11. Liu D.M., Wang Z.H., Liu L., Zhang X.M., Lou F.L.: Mol. Med. Rep. 8, 1453 (2013).

12. Ma X.L., Zhang F., Wang Y.X., He C.C., Tian K. et al.: Chem.-Biol. Interact. 254, 73 (2016).

13. Jung C.H., Kim Y., Kim M.S., Lee S., Yoo S.H.: Phytochem. Anal. 27, 140 (2016).

14. Kim E., Lim S.M., Kim M.S., Yoo S.H., Kim Y.: Nutrients 9, 1049 (2017). 
15. Nabekura T., Yamaki T., Ueno K., Kitagawa S.: Cancer Chemother. Pharmacol. 62, 867 (2008).

16. Zhang H., Matsuda H., Kumahara A., Ito Y., Nakamura S., Yoshikawa M.: Bioorg. Med. Chem. Lett. 17, 4972 (2007).

17. Wang Q., Matsuda H., Matsuhira K., Nakamura S., Yuan D., Yoshikawa M.: Biol. Pharm. Bull. 30, 388 (2007).

18. Kawamura M., Kagata M., Masaki E., Nishi H.: Pharmacol. Toxicol. 90, 106 (2002).

19. Nozawa K., Yamada M., Tsuda Y., Kawai K., Nakajima S.: Chem. Pharm. Bull. 29, 2689 (1981).

20. Ji H.J., Wang D.M., Hu J.F., Sun M.N., Li G. et al.: Eur. J. Pharmacol. 723, 259 (2014).
21. Zhu J.R., Tao Y.F., Lou S., Wu Z.M.: Acta Pharmacol. Sin. 31, 273 (2010.

22. Zhu Q.L., Li Y.X., Zhou R., Ma N.T., Chang R.Y. et al.: Pharm. Biol. 52, 1052 (2014).

23. Blanco-Suarez E., Hanley J.G.: J. Biol. Chem. 289, 4644 (2014).

24. Iijim T.H., Kensuke T., Sachie M.: Neurosci. Lett. 448, 268 (2008).

25. Budihardjo I., Oliver H., Lutter M., Luo X., Wang X.: Annu. Rev. Cell Dev. Biol. 15, 269 (1999).

26. Chan P.H.: J. Cereb. Blood Flow Metab. 21, 2 (2001).

27. Youle R.J. Strasser A.: Nat. Rev. Mol. Cell Biol. 9, 47 (2008).

Received: 8.07.2019 